\title{
Photic Regulation of Arylalkylamine $N$-Acetyltransferase Binding to 14-3-3 Proteins in Retinal Photoreceptor Cells
}

\author{
Nikita Pozdeyev, ${ }^{1}$ Carla Taylor, ${ }^{1}$ Rashidul Haque, ${ }^{1}$ Shyam S. Chaurasia, ${ }^{1}$ Amy Visser, ${ }^{1}$ Aamera Thazyeen, ${ }^{1}$ Yuhong Du, ${ }^{1}$ \\ Haian Fu, ${ }^{1}$ Joan Weller, ${ }^{3}$ David C. Klein, ${ }^{3}$ and P. Michael Iuvone ${ }^{1,2}$ \\ Departments of ${ }^{1}$ Pharmacology and ${ }^{2}$ Ophthalmology, Emory University School of Medicine, Atlanta, Georgia 30322, and ${ }^{3}$ Laboratory of \\ Neuroendocrinology, National Institute of Child Health and Human Development, National Institutes of Health, Bethesda, Maryland 20892-4480
}

14-3-3 proteins are a ubiquitous, highly conserved family of chaperone proteins involved in signal transduction, regulation of cell cycle, intracellular trafficking/targeting, cytoskeletal structure, and transcription. Although 14-3-3 proteins are among the most abundant proteins in the CNS, very little is known about their functional roles in the vertebrate retina. In the present study, we demonstrated that photoreceptors express 14-3-3 protein(s) and identified a 14-3-3 binding partner in photoreceptor cells, the melatonin-synthesizing enzyme arylalkylamine $N$-acetyltransferase (AANAT). Importantly, our data demonstrate that the binding of 14-3-3 to AANAT is regulated by light, with dramatic functional consequences. During the night in darkness, retinal AANAT is phosphorylated and forms a complex with 14-3-3 proteins with an apparent molecular weight of $\sim 90 \mathrm{kDa}$. Phosphorylation of AANAT facilitates the binding of enzyme to 14-3-3 proteins. Within the complex, AANAT is catalytically activated and protected from dephosphorylation and degradation. Light disrupts the AANAT/14-3-3 complex, leading to catalytic inactivation, dephosphorylation, and proteolytic degradation of the enzyme. In the presence of the proteasome inhibitor, lactacystin, light results in the formation of a high molecular weight complex ( $>150$ $\mathrm{kDa}$ ), which may represent an intermediate in the AANAT degradation process. These findings provide new insight into the roles of 14-3-3 proteins in photoreceptor cells and to the mechanisms controlling melatonin synthesis in the vertebrate retina.

Key words: arylalkylamine- $\mathrm{N}$-acetyltransferase; melatonin; 14-3-3 proteins; retina; photoreceptors; phosphorylation; light

\section{Introduction}

14-3-3 proteins are a family of dimeric binding proteins that interact with a wide variety of ligand proteins to regulate signal transduction, cell cycle, intracellular trafficking/targeting, cytoskeletal structure, enzyme activity, and transcription (Fu et al., 2000; Aitken et al., 2002). 14-3-3 proteins are very abundant in the CNS (Moore and Perez, 1967), estimated to constitute $~ 1 \%$ of soluble protein in brain. However, the roles of 14-3-3 in retinal function are poorly understood. In Drosophila, 14-3-3 proteins have been implicated in photoreceptor development (Kockel et al., 1997). In vertebrate photoreceptors, the only known ligand of 14-3-3 is the $\mathrm{G}_{\beta \gamma}$ interacting protein phosducin (Nakano et al., 2001). Here, we examined the light-regulated interaction of 14-3-3 with another photoreceptor protein, the melatonin-synthesizing enzyme arylalkylamine $N$-acetyltransferase (AANAT; EC 2.3.1.87).

Melatonin is a neurohormone produced by retinal photoreceptors and pinealocytes (Lerner et al., 1958; Gern and Ralph, 1979; Falcon and Collin, 1991; Cahill and Besharse, 1992; Bernard et al., 1997; Niki et al., 1998). Pineal-derived melatonin

Received Dec. 8, 2005; revised July 31, 2006; accepted Aug. 1, 2006.

This study was supported in part by National Institutes of Health Grants R01 EY04864 and P30 EY06360. C.T. participated in the Summer Undergraduate Research Experience (SURE), supported by Howard Hughes Medical Institute Grant 52003727.

Correspondence should be addressed to P. Michael luvone, Department of Pharmacology, Emory University School of Medicine, 1510 Clifton Road, Atlanta, GA 30322. E-mail: miuvone@pharm.emory.edu.

DOI:10.1523/JNEUROSCI.1384-06.2006

Copyright $\odot 2006$ Society for Neuroscience $\quad$ 0270-6474/06/269153-09\$15.00/0 functions as a circulating hormone. In contrast, retinal melatonin functions locally as a neuromodulator, regulating circadian aspects of adaptation and physiology in the retinal network (Besharse et al., 1988; Green and Besharse, 2004; Iuvone et al., 2005). The avian retina has been used extensively to study circadian control of melatonin synthesis and its receptor-mediated actions. The key regulatory step in melatonin biosynthesis is catalyzed by AANAT (Klein et al., 1997), which exhibits a daily rhythm of expression and activity that is regulated by a circadian clock. In the chicken retina, AANAT expression and circadian clocks are both intrinsic properties of photoreceptor cells (for review, see Iuvone et al., 2005). Rhythmic aanat gene expression is thought to be controlled by cycling components of the clock (Chong et al., 2000). However, AANAT activity and mRNA do not change in parallel under all circumstances. For example, in diurnal lighting, the amplitude of the activity rhythm is higher than that of mRNA abundance. In addition, light exposure at night rapidly suppresses AANAT protein and activity without altering AANAT mRNA levels (Bernard et al., 1997; Iuvone et al., 2002). These discrepancies indicate the existence of lightregulated posttranscriptional and/or posttranslational mechanisms in the control of AANAT activity in photoreceptor cells. Light acts directly on retinal photoreceptors to decrease AANAT activity and protein by a mechanism involving proteasomal proteolysis (Iuvone et al., 2002).

AANAT forms a complex with 14-3-3 proteins (Obsil et al., 2001). Roseboom et al. (1994) showed that 14-3-3 copurified 
with AANAT from sheep pineal glands. Subsequent studies demonstrated that phosphorylation-dependent binding to 14-3-3 proteins activates AANAT and protects it from proteolytic degradation (Ganguly et al., 2001, 2005; Zheng et al., 2003, 2005). In this study, we investigated the role of 14-3-3 complex formation in the photic regulation of AANAT in chicken photoreceptor cells. The findings of this study provide new insights into the control of AANAT and the roles of 14-3-3 in vertebrate photoreceptor cells.

\section{Materials and Methods}

Animals. Male White Leghorn chickens (Gallus domesticus) were acquired (HyLine International, Covington, GA) on the day of hatching and maintained in heated brooders in a $12 \mathrm{~h}$ light/dark cycle, with lights on from zeitgeber time (ZT) 0 to ZT12. They were used in experiments at $2-3$ weeks of age. All manipulations on chickens and tissues under conditions of darkness were performed under dim red light (number 92 filter; Eastman Kodak, Rochester, NY). Chickens were killed by decapitation, and retinas were rapidly dissected and frozen on dry ice. The experimental protocols met the guidelines of the National Institutes of Health Guide for the Care and Use of Laboratory Animals and were approved by the Institutional Animal Care and Use Committee of Emory University.

Lactacystin (Calbiochem, La Jolla, CA) was dissolved in $\mathrm{H}_{2} \mathrm{O}$ and administered intraocularly to chickens anesthetized with halothane; 25 nmol were delivered into vitreous cavity of the right eye by injection through a 30 gauge syringe needle in a volume of $10 \mu \mathrm{l}$. The left (control) eye of the chicken was injected with water. All injections were performed at ZT17.

For light treatment, chickens were put in a cage made from transparent plastic with a cool white fluorescent lamp mounted on top at ZT18. Light irradiance on the bottom of the cage was $\approx 100 \mu \mathrm{W} / \mathrm{cm}^{2}$. Retinas were dissected after 5-60 min of the light treatment. Control animals were kept and dissected in darkness.

AANAT assay and kinetic analysis. Retinas were homogenized in $0.1 \mathrm{M}$ ammonium acetate buffer containing $1.4 \mathrm{~mm}$ acetyl coenzyme A, $10 \mathrm{~mm}$ dithiothreitol, and $10 \%$ glycerol, $\mathrm{pH} 6.8 ; 25 \mu \mathrm{l} /$ retina of protease inhibitor mixture (Sigma, St. Louis, MO) was added to homogenization solution. After centrifugation at $15,000 \times g$ for $10 \mathrm{~min}$, the supernatant fraction was used for AANAT activity measurement or gel chromatography experiments.

AANAT activity was assayed by measuring the catalytic formation of $\mathrm{N}$-acetyltryptamine from tryptamine and acetyl coenzyme A with quantification of the reaction product by reversed-phase ion-pair HPLC with fluorescence detection (Thomas et al., 1990). Unless noted otherwise, sample was incubated in the presence of $1 \mathrm{~mm}$ tryptamine and $1.4 \mathrm{~mm}$ acetyl coenzyme A for $15 \mathrm{~min}$ at $37^{\circ} \mathrm{C}$. $\mathrm{N}$-Acetyltryptamine was extracted from the reaction mixture by toluene-acidified isoamyl alcohol (99:1, $\mathrm{v} / \mathrm{v})$. Organic phase was dried under the stream of nitrogen. Residue was resuspended in HPLC mobile phase and injected into the HPLC column. Separation was performed on a Whatman (Clifton, NJ) PartiSphere $\mathrm{C}_{18}$ $5 \mu \mathrm{m}$ reversed phase analytical column $(110 \times 4.7 \mathrm{~mm})$. The mobile phase consisted of $50 \mathrm{~mm}$ phosphoric acid, $1 \mathrm{~mm}$ sodium octylsulfate, and $28 \%$ methanol (v/v) adjusted to $\mathrm{pH} 3.0$ with sodium hydroxide and was pumped at a flow rate of $1.5 \mathrm{ml} / \mathrm{min}$. $N$-Acetytryptamine was detected by fluorescence (excitation, $283 \mathrm{~nm}$; emission, $352 \mathrm{~nm}$ ).

For measuring AANAT activity in fast protein liquid chromatography (FPLC) fractions, a modification of the method was used. The enzymatic reaction was stopped by addition of perchloric acid ( $0.2 \mathrm{~N}$ final concentration) and, after centrifugation at $15.000 \times g$ for $10 \mathrm{~min}$, supernatant was directly injected into the HPLC system.

For AANAT $K_{\mathrm{m}}$ and $V_{\max }$ measurements, enzymatic activity was estimated in the presence of a range of tryptamine concentrations from $1 \mu \mathrm{M}$ to $1 \mathrm{~mm}$. The experimental data were fit with the Michaelis-Menten equation using SigmaPlot 8.0 software (Systat Software, Point Richmond, CA) nonlinear regression analysis.

Protein concentration was measured by the method of Lowry et al. (1951), using bovine serum albumin as standard.
FPLC. Soluble proteins from chicken retinas were separated by gelpermeation chromatography using a Pharmacia Biotech FPLC system and Superdex 75 10/300 GL or Superdex 200 10/300 GL columns (Amersham Biosciences, Piscataway, NJ). Before injection, samples were filtered through $13 \mathrm{~mm}$ polysulfone filter devices $(0.2 \mu \mathrm{m}$ pore size; Whatman). Unless noted otherwise, the mobile phase consisted of $0.1 \mathrm{M}$ ammonium acetate, $10 \mathrm{~mm}$ dithiothreitol, 10\% glycerol, pH 6.8 (FPLC buffer). For chromatographic analysis, $0.5 \mathrm{ml}$ fractions were collected and $67.5 \mu \mathrm{l}$ of fraction was immediately combined with $7.5 \mu \mathrm{l}$ of $14 \mathrm{~mm}$ acetyl coenzyme A to stabilize AANAT (Binkley et al., 1976; Namboodiri et al., 1979). In some experiments, phosphate buffer $(0.25 \mathrm{M}, \mathrm{pH} 6.5)$ was used for FPLC to promote dissociation of AANAT/14-3-3 complex.

Western blotting of AANAT and 14-3-3 proteins. FPLC fraction $(350 \mu \mathrm{l})$ was concentrated using Microcon centrifugal filter devices (Millipore, Billerica, MA), and proteins were denatured by 5 min of boiling and separated on $10 \%$ Bis-Tris Criterion XT precast gels (Bio-Rad, Hercules, CA).

Retinal samples were homogenized in FPLC buffer containing $2 \mathrm{~mm}$ PMSF and protease inhibitor mixture (Sigma). When anti-phosphoAANAT antiserum was used, the homogenization solution was supplemented with $1 \mathrm{~mm} \mathrm{NaF}$ and phosphatase inhibitor mixtures 1 and 2 from Sigma (unless specified otherwise).

After semi-dry transfer of proteins to polyvinylidene difluoride membrane, AANAT enzyme was detected by rabbit polyclonal antibody 2992 directed against chicken AANAT $_{1-21}$ (Iuvone et al., 2002). Phosphorylated AANAT was detected with a phosphospecific rabbit polyclonal antibody 3352 directed against rat $\mathrm{pT}^{31}$-AANAT ${ }_{22-37}$ (Ganguly et al., 2001, 2005). 14-3-3 was detected with a mouse monoclonal antibody 7A3, which detects most isoforms of 14-3-3 (R. Subramanian, H. Fu, and A. Levey, unpublished observation). Band densities were quantified using Kodak Molecular Imaging software.

Retinal AANAT coimmunoprecipitation with 14-3-3 antibody. The chicken retinas were homogenized in $0.5 \mathrm{ml}$ of $0.1 \mathrm{M}$ ammonium acetate buffer, pH 7.4, containing $10 \mathrm{~mm}$ dithiothreitol, $1.4 \mathrm{~mm}$ acetyl-CoA, 10 $\mu \mathrm{M}$ lactacystin, and $25 \mu \mathrm{l}$ of $10 \times$ protease inhibitor mixture (Sigma). After centrifugation at $15,000 \times g$ for $10 \mathrm{~min}$, the supernatant was precleaned with $50 \mu \mathrm{l}$ of a $50 \%$ slurry of protein G Sepharose 4 Fast Flow (Amersham Biosciences, Uppsala, Sweden) for $1 \mathrm{~h}$ at $+4^{\circ} \mathrm{C}$. The supernatant was incubated with $20 \mu \mathrm{l}$ of 14-3-3 antibody (T-16; Santa Cruz Biotechnology, Santa Cruz, CA) or normal IgG for $2 \mathrm{~h}$ at $+4^{\circ} \mathrm{C}$ with mixing. The antibody/protein complexes were bound to $50 \mu \mathrm{l}$ of a $50 \%$ slurry of protein $\mathrm{G}$ Sepharose 4 Fast Flow beads during a $2 \mathrm{~h}$ incubation at $+4^{\circ} \mathrm{C}$. The beads were washed three times with ammonium acetate buffer and boiled in the presence of XT loading buffer $(1 \times$ final concentration; Bio-Rad). The samples were analyzed by Western blotting with AANAT protein detected with antibody 2992 directed against chicken $\operatorname{AANAT}_{1-21}$ (1:200) (Iuvone et al., 2002). A coimmunoprecipitation was also performed from the FPLC fraction corresponding to Peak 2 (see Fig. 2). The FPLC separation of proteins from the dark-adapted nighttime retinas was performed as described above, except that the mobile phase was modified to make it compatible with the immunoprecipitation protocol (0.1 $\mathrm{M}$ ammonium acetate, $10 \mathrm{~mm}$ dithiothreitol, $\mathrm{pH} 7.4) ; 200 \mu \mathrm{l}$ of fraction was supplemented with $1.4 \mathrm{~mm}$ acetyl-CoA and incubated with $20 \mu \mathrm{l}$ of $14-3-3$ antibody or normal IgG overnight at $+4^{\circ} \mathrm{C}$. The antibody/protein complexes were precipitated, separated, and detected as described above for retinal extracts.

Detection of 14-3-3 proteins in photoreceptor cells. Photoreceptor cells were isolated by laser capture microdissection according to a procedure described previously (Haque et al., 2002; Chaurasia et al., 2005). Photoreceptor cells were dissected from five $5 \mu \mathrm{m}$ sections of chicken retina using a PixCell IIe laser capture microdissection system (Arcturus Engineering, Mountain View, CA) (see Fig. 3A-C). The film containing dissected tissue was incubated in $20 \mu \mathrm{l}$ of T-Per tissue protein extraction reagent (Pierce Biotechnology, Rockford, IL) for $20 \mathrm{~min}$ at $+4^{\circ} \mathrm{C}$. The sample was supplemented with XT loading buffer $(1 \times$ final concentration; Bio-Rad), boiled for $5 \mathrm{~min}$, and analyzed by Western blotting with anti-14-3-3 mouse monoclonal antibody 7A3, as described above.

Investigation of the AANAT/14-3-3 complex with synthetic peptides. All peptides were synthesized by the Emory University Microchemical Facil- 


\begin{tabular}{lll}
\hline Name & Sequence & Description \\
\hline NCTRL & $\mathrm{NH}_{2}$-GTQRPRRPSHERFCLA-COOH & Scrambled peptide corresponding to N-terminal AANAT-specific peptide \\
NAT22-37 & $\mathrm{NH}_{2}$-PGRQRRHTLPASEFRC-COOH & AANAT-specific peptide containing nonphosphorylated N-terminal phosphorylation site \\
pNAT22-37 & $\mathrm{NH}_{2}$-PGRQRRH(p)TLPASEFRC-COOH & AANAT-specific peptide containing phosphorylated N-terminal phosphorylation site \\
CCTRL & $\mathrm{NH}_{2}$-SHAGFRGRNCM-COOH & Scrambled peptide corresponding to C-terminal AANAT-specific peptide \\
NAT195-205 & $\mathrm{NH}_{2}$-GHAFMRRNSGC-COOH & AANAT-specific peptide containing nonphosphorylated C-terminal phosphorylation site \\
pNAT195-205 & $\mathrm{NH}_{2}$-GHAFMRRN(p)SGC-COOH & AANAT-specific peptide containing phosphorylated C-terminal phosphorylation site \\
\hline
\end{tabular}
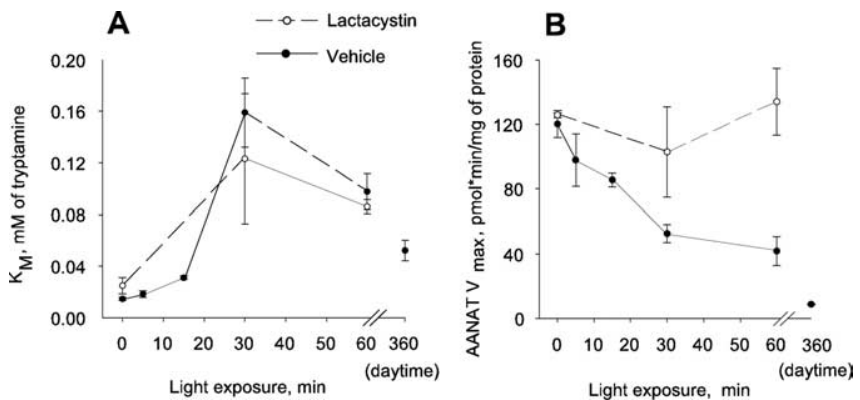

Figure 1. Light regulates AANAT apparent $K_{\mathrm{m}}$ and $V_{\max }$ in the chicken retina. Apparent $K_{\mathrm{m}}$ and $V_{\max }$ of AANAT were measured in chicken retinas dissected at ZT18 in darkness or after 5-60 min of light exposure. Daytime values were obtained at ZT6. Animals were injected intraocularly with $10 \mu$ l of water or 2.5 mm lactacystin. $K_{\mathrm{m}}$ and $V_{\text {max }}$ data are presented in $A$ and $B$, respectively. For $K_{\mathrm{m}}$ measurements, two-way ANOVA indicates a significant effect of light $(p<0.001)$ but not of lactacystin ( $p=0.407)$. For $V_{\text {max }}$ measurements, there were significant effects of both light $(p<0.05)$ and lactacystin $(p<0.01)$ and a significant interaction of light and lactacystin $(p=0.05)$. Values are means $\pm \operatorname{SEM}(n=4)$.

ity. Peptides were purified by HPLC, and their identity was verified by MALDI mass spectrometric analysis. Sequences of peptides are presented in Table 1.

Statistical analysis. The effect of light and lactacystin in vivo on AANAT kinetic parameters was assessed by two-way ANOVA. One-way ANOVA was used for the analysis of FPLC experimental results. Repeatedmeasures ANOVA (RM-ANOVA) was used when applicable. When ANOVA revealed the existence of statistically significant differences between groups, pair-wise comparisons were done by Student-NewmanKeuls test. When an experiment had only two groups to compare, Student's $t$ test was used.

\section{Results}

Light regulates apparent $K_{\mathrm{m}}$ and $V_{\max }$ of AANAT in the chicken retina

Exposing chickens to light in the middle of the night increased the apparent $K_{\mathrm{m}}$ of AANAT for tryptamine by $\sim 10$-fold (Fig. $1 \mathrm{~A}$ ) (two-way ANOVA; $p<0.001$ ). The $K_{\mathrm{m}}$ began to increase within 5 min of exposure to light and reached a maximum at $\sim 30 \mathrm{~min}$. Treatment with lactacystin, a specific inhibitor of proteasomal proteolysis, did not affect the $K_{\mathrm{m}}$ of the enzyme. The apparent $K_{\mathrm{m}}$ in the middle of the day (in light) was intermediate when compared with the $K_{\mathrm{m}}$ values of the enzyme from nighttime darkadapted retinas and after acute exposure to light.

The $V_{\max }$ of retinal AANAT decreased by $\sim 30 \%$ after $15 \mathrm{~min}$ of light exposure (Fig. 1B) (two-way ANOVA; $p<0.05$ ) and continued to decline thereafter. Lactacystin treatment antagonized the effect of light on AANAT $V_{\max }(p<0.05)$. The $V_{\max }$ of AANAT was very low in daytime samples, consistent with the low levels of retinal melatonin in the daytime (Hamm and Menaker, 1980).

Retinal AANAT occurs in three fractions with distinct apparent molecular weights

When soluble proteins from nighttime dark-adapted retina (ZT18) were separated by gel permeation chromatography on a
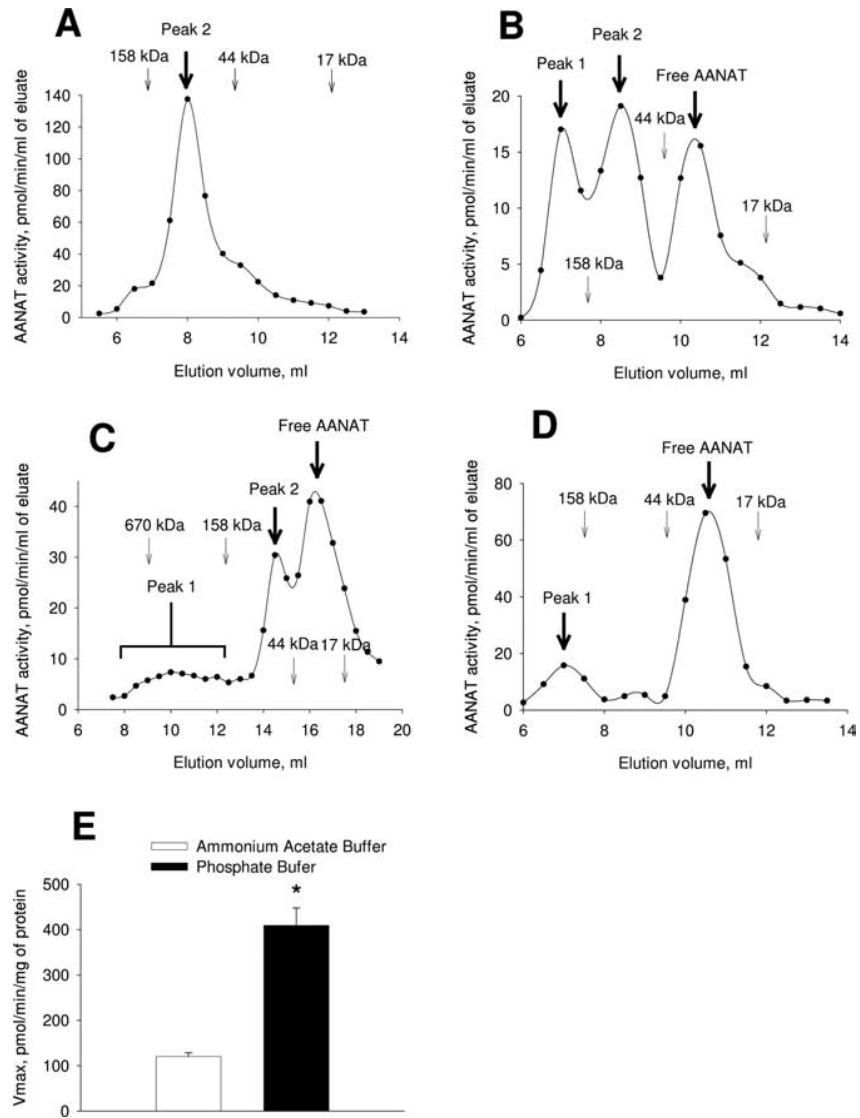

Figure 2. Three peaks of AANAT activity from retinal proteins separated by gel permeation chromatography. Chicken retinas were homogenized in FPLC buffer and centrifuged, and the supernatant fraction was subjected to gel permeation chromatography on Superdex 75 10/300 GL column, unless stated otherwise. AANAT activity was measured in FPLC fractions as described in Materials and Methods. The thin arrows represent locations of molecular weight standards. A, Nighttime dark-adapted retina (ZT18). B, Lactacystin-treated nighttime retina after $45 \mathrm{~min}$ of light exposure. C, FPLC profile of AANAT from lactacystin-treated light-exposed retina after separation on Superdex 200 10/300 GL column. D, Lactacystin-treated nighttime retina after 45 min of light exposure separated on Superdex $7510 / 300 \mathrm{GL}$ in $0.25 \mathrm{~m}$ phosphate buffer, pH 6.5 . $\boldsymbol{E}$, Homogenization of retinas in phosphate buffer, which dissociates the AANAT/14-3-3 complex, causes a 3.4-fold increase in AANAT $V_{\max }{ }^{(*} p<0.001$; Student's $t$ test). Values are means $\pm \operatorname{SEM}(n=4)$.

Superdex 75 10/300 GL column, most of the activity was eluted as a peak with apparent molecular weight of $\sim 80-100 \mathrm{kDa}$ (Fig. $2 A$ ). The predicted molecular weight of chicken AANAT is 23 $\mathrm{kDa}$ (Bernard et al., 1997). Thus, AANAT in dark-adapted retina appears to exist in a protein complex.

In extracts of lactacystin-treated retinas exposed to light for 45 min, AANAT activity in the $\sim 80-100 \mathrm{kDa}$ peak declined, and two more peaks with significant AANAT activity became distinguishable (Fig. $2 \mathrm{~B}$ ). One of these has a high apparent molecular weight $(>150 \mathrm{kDa})$. The molecular weight of the third peak corresponded to free monomeric AANAT, $\sim 23 \mathrm{kDa}$. 
In an attempt to better characterize AANAT in the first peak from lighttreated retinas, proteins were separated on a Superdex 200 10/300 GL column. However, a distinct peak 1 was not obtained, although peaks 2 and 3 were well resolved (Fig. 2C). Rather than being associated with a single size fraction, AANAT activity corresponding to peak 1 was recovered in a large range of fractions (150 to $>670$ $\mathrm{kDa})$, suggesting that peak 1 is not homogenous.

Several buffers were used for the mobile phase in gel permeation chromatography; it was found that $0.25 \mathrm{M}$ potassium phosphate buffer, $\mathrm{pH}$ 6.5, commonly used for AANAT enzyme assay (Hamm and Menaker, 1980; Thomas et al., 1990), caused dissociation of the $\sim 80-100 \mathrm{kDa}$ AANAT complex but did not affect peak 1 (Fig. 2D).

The apparent $K_{\mathrm{m}}$ for tryptamine of AANAT in peak 2 was 15-20 times lower than the $K_{\mathrm{m}}$ of the free enzyme (peak 3 ) or the high molecular weight AANAT fraction (peak 1) (Table 2). We next compared the $V_{\max }$ of AANAT in extracts of darkadapted retinas prepared in ammonium acetate buffer, which preserves the AANAT complex from peak 2, or in phosphate buffer, to dissociate it (Fig. 2E). The $V_{\max }$ in phosphate buffer was 3.4-fold higher than that in ammonium acetate, suggesting that the free form of the enzyme has a higher $V_{\max }$ than the enzyme in peak 2 .

\section{Peak 2 contains AANAT complexed with 14-3-3}

Recombinant phosphorylated ovine AANAT forms a complex with 14-3-3 $\zeta$ that migrates in gel filtration chromatography with an apparent molecular weight of $\sim 90 \mathrm{kDa}$ (Ganguly et al., 2001), similar to peak 2 from retinal extracts. Chicken AANAT, like ovine AANAT, contains a consensus 14-3-3 binding motif with an embedded protein kinase A (PKA) phosphorylation site, RRHpTLPAS (Bernard et al., 1997). We therefore investigated whether 14-3-3 proteins are expressed in chicken photoreceptor cells and whether peak 2 represents an AANAT/14-3-3 complex.

We identified 14-3-3 by Western blot analysis of proteins from photoreceptor cells isolated by laser capture microdissection of frozen sections of retina (Fig. 3). The observation that 14-3-3 proteins are detectable in such a small amount of tissue (photoreceptors from five 5 - $\mu \mathrm{m}$-thick sections were pooled together) indicates that 14-3-3 proteins are abundant in photoreceptors and are likely to play a major role in photoreceptor physiology.

To determine whether chicken retinal AANAT binds directly to 14-3-3, coimmunoprecipitation experiments were conducted. Figure $4, A$ and $B$, shows that AANAT was immunoprecipitated from extracts of dark-adapted retina and the FPLC fraction corresponding to peak 2 of AANAT activity by rabbit anti-14-3-3 but not by normal rabbit IgG.

To further confirm that peak 2 represents the AANAT/14-3-3 complex, extracts from dark-adapted retinas were incubated for
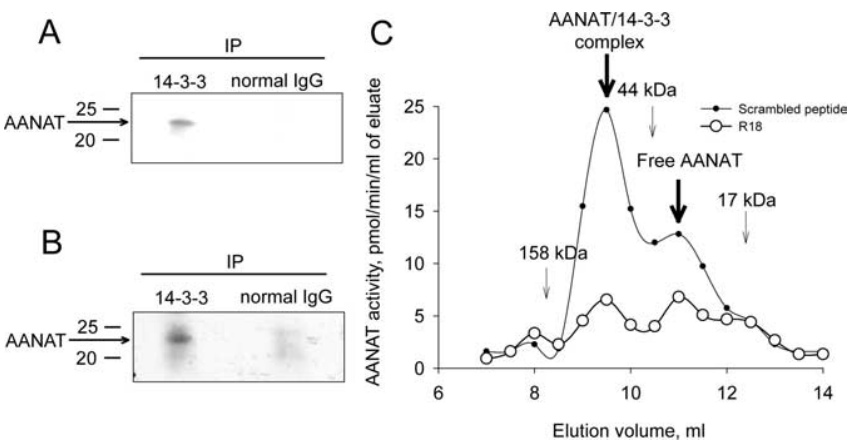

Figure 4. AANAT and 14-3-3 proteins form a complex in chicken retina. $\boldsymbol{A}, \boldsymbol{B}$, AANAT was coimmunoprecipitated from protein extract of dark-adapted retina $(\boldsymbol{A})$ and FPLC fraction corresponding to peak 2 of AANAT activity $(\boldsymbol{B})$ with rabbit anti-14-3-3 but not with normal rabbit lgG. AANAT was detected with rabbit antibody 2992, directed against chicken AANAT ${ }_{1-21}$ (1:200) (luvone et al., 2002). Molecular weight standards are shown on the left. These experiments were replicated twice with similar results. $C$, Incubation with R18 peptide causes dissociation of $\sim 80-100$ kDa peak and reduced total enzyme activity. Protein extracts from nighttime darkadapted retinas were incubated in the presence of $10 \mu \mathrm{m}$ lactacystin, $20 \mu \mathrm{m}$ R18, or scrambled peptide for $16 \mathrm{~h}$ at $+4^{\circ} \mathrm{C}$. Separation was performed on a Superdex 75 10/300 GL column. Chromatographic profiles representative of results from five separate experiments are shown.

16 h with R18 (10 $\mu \mathrm{M})$, a peptide that specifically inhibits $14-3-3$ interactions by occupying the 14-3-3 binding groove (Petosa et al., 1998; Wang et al., 1999). Incubation of retinal proteins with R18 peptide before FPLC separation caused significant degradation of the $\sim 80-100 \mathrm{kDa}$ AANAT complex, as evidenced by the large decrease of AANAT activity in peak 2 (Fig. $4 C)(n=5$ 


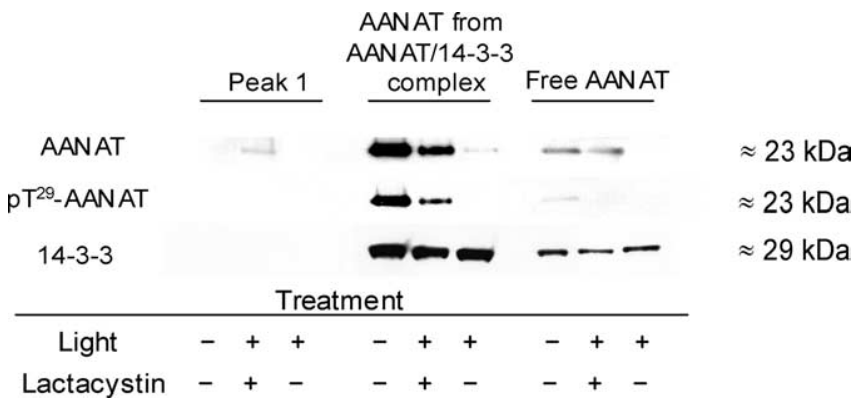

Figure 5. Western blot analysis of AANAT and 14-3-3 proteins from FPLC separations. Proteins from nighttime dark-adapted (ZT18), light-treated lactacystin-injected (45 min of light at $\mathrm{ZT} 18,25 \mathrm{nmol}$ of lactacystin/eye), and light-treated vehicle-injected retinas were separated by FPLC on a Superdex 75 10/300 GL column. Two retinas were used for each separation. Fractions corresponding to the three peaks of AANAT activity were concentrated and subjected to Western blot analysis as described in Materials and Methods. The same blot was labeled with antibodies specific to pan-AANAT (antibody 2992, 1:200), phospho-AANAT ( $\mathrm{pT}^{29}$-AANAT; antibody $3352,1: 100)$, and 14-3-3 proteins (7A3, 1:5000). The approximate molecular weights of the bands are shown. This experiment was repeated twice with similar results.

experiments; $p<0.005$; paired Student's $t$ test). In addition, phosphorylated $\mathrm{pT}^{29}$-AANAT and 14-3-3 protein coelute in peak 2 from dark-adapted retinal extracts (Fig. 5). Coupled with the coimmunoprecipitation results (Fig. $4 B$ ), these findings indicate that peak 2 represents a complex of phosphorylated AANAT with 14-3-3 proteins. It is noteworthy that a small amount of $\mathrm{pT}^{29}$-AANAT was observed in the monomeric peak from dark-adapted retinas, indicative of a dynamic equilibrium between the 14-3-3 bound and unbound forms of phosphorylated AANAT.

\section{The effect of light treatment on retinal AANAT forms}

Exposure to $45 \mathrm{~min}$ of light at night caused nearly complete degradation of AANAT protein and loss of activity in all three fractions (Figs. 5 and 6, respectively). The loss of AANAT protein and activity was partially prevented by pretreatment with lactacystin. Lactacystin treatment does not affect the AANAT-14-3-3 complex/free AANAT ratio (Fig. $6 F$ ). These results are consistent with the results of total AANAT $V_{\max }$ measurements in retinal supernatant (Fig. 1) and provide an explanation for light-induced changes in AANAT $K_{\mathrm{m}}$ (i.e., light causes dissociation of the AANAT/14-3-3 complex). Observations presented here (Fig. 2 E, Table 2) and in the literature (Ganguly et al., 2001) indicate that free AANAT has a higher apparent $K_{\mathrm{m}}$ and $V_{\max }$ than does 14-33-bound AANAT.

Light treatment caused a $\sim 2.5$-fold increase in the AANAT activity associated with the high molecular weight peak 1 but only when proteasomal proteolysis was inhibited (Fig. 6B).

\section{The effect of light treatment on retinal}

\section{AANAT phosphorylation}

Western blot analysis was used to estimate the $\mathrm{pT}^{29}$-AANAT/ total AANAT ratio in dark-adapted and light-treated chicken retinas (Fig. 7). Retinas were homogenized in ammonium acetate buffer containing a phosphatase inhibitor mixture to minimize AANAT/14-3-3 complex dissociation and enzyme dephosphorylation. Light treatment $(30-45 \mathrm{~min})$ decreased the proportion of AANAT phosphorylated on Thr-29 by 60\%; normalized phosphorylated AANAT/AANAT ratio was $1.00 \pm 0.24$ and $0.40 \pm$ 0.07 for dark adapted and light exposed retinas, respectively $(n=$ $7 ; p<0.05$; Student's $t$ test). This result is indicative of enhanced dephosphorylation during light exposure.
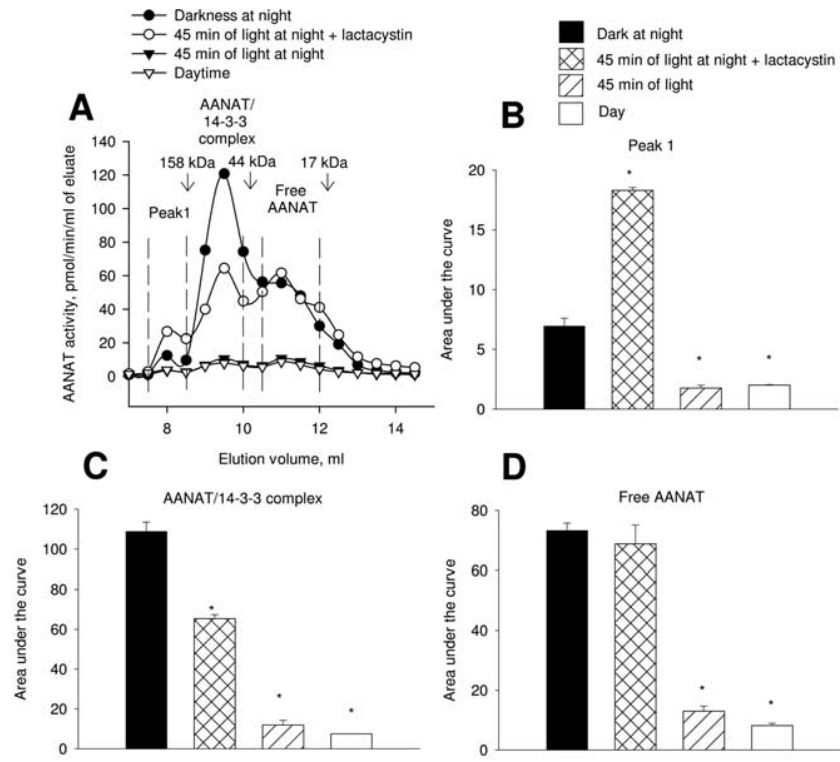

E

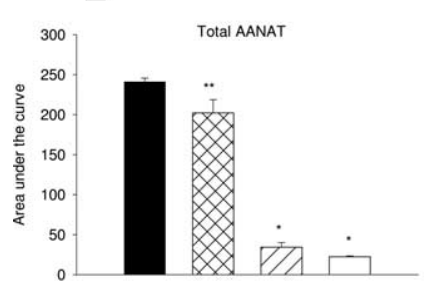

$\mathbf{F}$

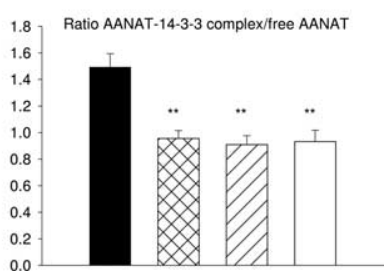

Figure 6. The effect of light treatment on AANAT activity peaks in the retina. Proteins from nighttime dark-adapted (ZT18), light-treated lactacystin-injected (45 min of light at ZT18, 25 nmol of lactacystin/eye), light-treated vehicle-injected, and daytime (ZT6) retinas were separated by FPLC on a Superdex 75 10/300 GL column, and AANAT activity was measured in fractions. A, Representative FPLC profiles are shown. Vertical dashed lines define the limits for calculating the area under the curve for each AANAT activity peak. The thin arrows indicate the location of molecular weight standards. $\boldsymbol{B}-\boldsymbol{F}$, The effect of light and lactacystin treatments on AANAT forms, total AANAT activity, and AANAT-14-3-3 complex/free AANAT ratio. ${ }^{*} p<0.001$; ${ }^{* *} p<0.05$; Student-Newman-Keuls test. Values are means \pm SEM $(n=3)$.

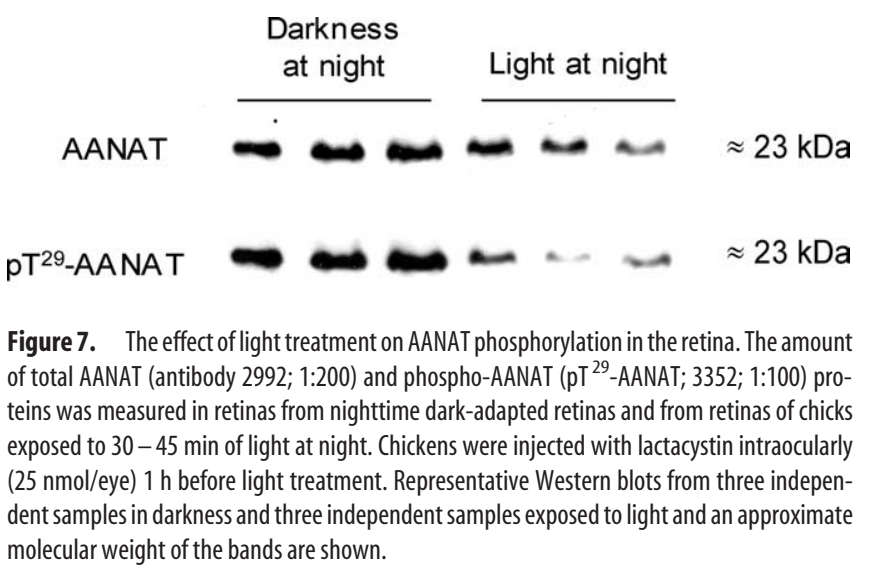

14-3-3 proteins protect AANAT from dephosphorylation and degradation

Supernatant fractions from dark-adapted retinas were either denatured in SDS sample buffer immediately after centrifugation or were incubated for $1 \mathrm{~h}$ at $4^{\circ} \mathrm{C}$ with or without $\mathrm{R} 18$ peptide, which dissociates the AANAT/14-3-3 complex (Fig. 4), before proceeding with Western blot analysis. Phosphatase inhibitors were not 


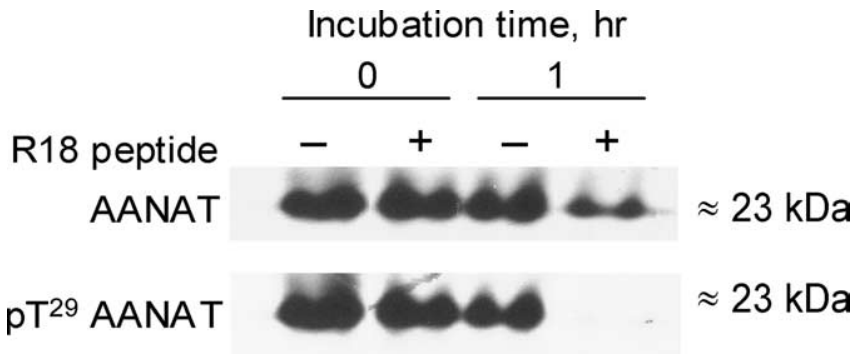

Figure 8. Binding to 14-3-3 proteins protects AANAT from degradation and dephosphorylation. Nighttime dark-adapted retinas were homogenized in ammonium acetate buffer not supplemented with phosphatase inhibitors. After centrifugation at $15,000 \times g$ for $10 \mathrm{~min}, \mathrm{R} 18$ peptide $(50 \mu \mathrm{m})$ or vehicle was added to supernatant fractions. The samples were either immediately denatured in SDS sample buffer or incubated for $1 \mathrm{~h}$ at $+4^{\circ} \mathrm{C}$ before denaturation. The relative abundance of AANAT and $\mathrm{pT}^{29}$-AANAT was assessed by Western blot analysis. Dissociation of AANAT/14-3-3 complex by R18 caused a reduction in total AANAT and virtually complete disappearance of $\mathrm{pT}^{29}$-AANAT. The approximate molecular weights of the bands are shown. This experiment was repeated twice with similar results.

added to homogenization solutions in this experiment to allow dephosphorylation of AANAT to occur. In control samples, AANAT protein was mostly preserved during the incubation. In contrast, AANAT protein decreased by 50\% when the AANAT/ 14-3-3 complex was dissociated (Fig. 8). This is consistent with previous findings that 14-3-3 proteins protect AANAT from degradation (Zheng et al., 2003, 2005). Similarly, $\mathrm{pT}^{29}$-AANAT immunoreactivity was preserved during the incubation in control samples but completely disappeared in the samples incubated with R18. Similar results were obtained when the AANAT/14-3-3 complex was dissociated with phosphate buffer (data not shown). Thus, binding to $14-3-3$ proteins protects AANAT from dephosphorylation.

\section{PKA phosphorylation sites Thr-29 and Ser-203 play a role in AANAT binding to $14-3-3$ proteins}

The role of Thr-29 and Ser-203 was investigated using phosphorylated and unphosphorylated AANAT peptides containing these sequences (Table 1). These two sequences correspond to those in ovine AANAT that mediate binding to 14-3-3 (Ganguly et al., 2001, 2005; Zheng et al., 2003, 2005).

Incubation of extracts of dark-adapted retina with the $\mathrm{N}$-terminal $\mathrm{pT}^{29}$-AANAT22-37 peptide caused dissociation of AANAT/14-3-3 complex and enzyme degradation (Fig. 9A) (one-way RM-ANOVA; $p<0.01$; Student-Newman-Keuls, $p<$ $0.05)$. Neither the unphosphorylated $\mathrm{N}$-terminal peptide nor the scrambled peptide had a statistically significant effect. In contrast, incubation with C-terminal $\mathrm{pS}^{203}$-AANAT195-205 alone had no effect on enzyme activity associated with the AANAT/143-3 peak but elicited small decreases in the activity of the free AANAT peak and in total AANAT activity (Fig. 9B) (one-way RM-ANOVA, $p<0.01$; Student-Newman-Keuls, $p<0.05$ ). Because the experiment with C-terminal AANAT peptides did not clarify the role of C-terminal phosphorylation site in enzyme binding to 14-3-3, we tested different combinations of phosphopeptides and nonphosphopeptides studying the cumulative effect of Thr-29 and Ser-203 phosphorylation on 14-3-3 binding (Fig. 9C). The effect on the AANAT/14-3-3 complex and enzyme degradation was most pronounced in the presence of both phosphorylated peptides (one-way RM-ANOVA, $p<0.01$; StudentNewman-Keuls, $p<0.05$ ).
cAMP and PKA favor the formation of the AANAT/14-3-3 complex

To study the role of the CAMP/PKA pathway in AANAT regulation, retinal extracts were incubated with ATP, PKA, and 8-BrcAMP in the presence of $\mathrm{Mg}^{2+}$. The incubation with ATP alone increased the enzyme activity in the free AANAT peak (Fig. $10 A, B$ ) (one-way RM-ANOVA, $p<0.05$; Student-NewmanKeuls, $p<0.05$ ). Exogenous catalytic subunit of PKA and 8-BrcAMP, apparently acting via endogenous PKA, both enhanced the association of AANAT with 14-3-3 proteins, resulting in an increase in the enzyme activity in the $\approx 80-100 \mathrm{kDa}$ peak (oneway RM-ANOVA, $p<0.05$; Student-Newman-Keuls, $p<0.05$ ).

\section{Discussion}

In chicken retina, AANAT is expressed primarily in photoreceptor cells (Bernard et al., 1997). The current study shows that 14-3-3 protein is also expressed in chicken photoreceptors and that AANAT in dark-adapted retina is bound to 14-3-3. This interaction is regulated by light and phosphorylation. Phosphorylation of AANAT in darkness promotes the association with 14-3-3, which decreases the $K_{\mathrm{m}}$ of the enzyme for substrate and protects the enzyme from dephosphorylation and degradation. Light exposure results in dephosphorylation of AANAT and dissociation from the complex with 14-3-3 proteins, thereby increasing the $K_{\mathrm{m}}$ of the enzyme. Dissociation of the complex leads to subsequent proteasomal proteolysis of AANAT.

A novel observation of the present study is that the apparent $K_{\mathrm{m}}$ of AANAT for substrate is regulated by light exposure. Light exposure at night increased the apparent $K_{\mathrm{m}} \sim 10$-fold. Previously, the available evidence indicated only that light-evoked physiological changes in AANAT activity in retina and pineal were attributable to protein degradation (Gastel et al., 1998; Schomerus et al., 2000; Zatz et al., 2000; Falcon et al., 2001; Iuvone et al., 2002). A physiological effect of 14-3-3 binding on the $K_{\mathrm{m}}$ of AANAT for amine substrates was inferred based on studies using expressed protiens (Obsil et al., 2001; Ganguly et al., 2001, 2005). The current results provide new insight on a mechanism whereby light can rapidly downregulate melatonin synthesis. Other related novel observations are that AANAT in retina exists in at least three physical states with distinct apparent molecular weights and kinetic characteristics and that these physical states exist in a dynamic equilibrium that is regulated by light. When retinal proteins were subjected to gel permeation chromatography, AANAT eluted in three peaks corresponding to the monomeric enzyme, an AANAT/14-3-3 complex, and a high molecular weight form of unknown identity. In darkness, most AANAT activity is found in the peak corresponding to the AANAT/14-3-3 complex, which decreases after light exposure. In lactacystintreated retinas, this effect of light is associated with corresponding increases in the high molecular weight peak of AANAT and in the ratio of free AANAT to AANAT/14-3-3 complex. The shift in equilibrium of these AANAT forms appears to be responsible for the light-evoked increase of apparent $K_{\mathrm{m}}$ for substrate observed in retinal homogenates; the apparent $K_{\mathrm{m}}$ values measured in the free AANAT peak and the high molecular weight AANAT peak are $>10$-fold higher than that measured in the AANAT/14-3-3 peak.

The apparent $K_{\mathrm{m}}$ of AANAT depends on the [AANAT/14-3-3 complex]/free AANAT ratio, which is affected by two factors: complex dissociation and free enzyme degradation. In darkness, the apparent $K_{\mathrm{m}}$ is low, because the ratio is high. Acute light at night dissociates the complex and increases the concentration of free AANAT, resulting in a high apparent $K_{\mathrm{m}}$. With prolonged 

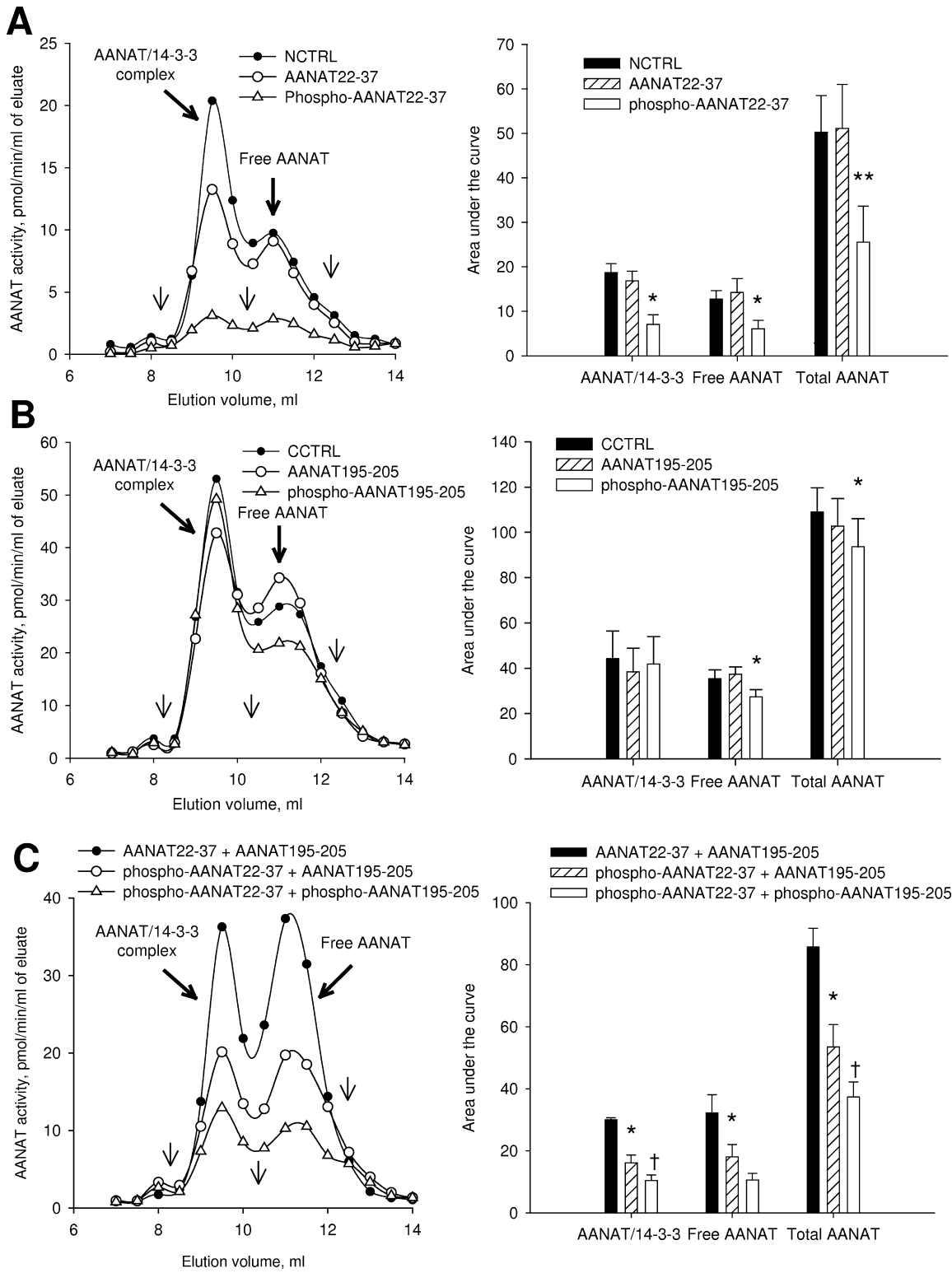

Figure 9. The role of Thr-29 and Ser-203 phosphorylation in the formation of AANAT/14-3-3 complex. After homogenization in ammonium acetate buffer and centrifugation at 15,000 $\times g$ for 10 min supernatant fractions from dark-adapted nighttime retinas were incubated in the presence of $100 \mu \mathrm{m}$ phosphorylated and nonphosphorylated AANAT-specific peptides (Table 1) for $16 \mathrm{~h}$ at $+4^{\circ} \mathrm{C}$ and separated by FPLC on a Superdex $7510 / 300 \mathrm{GL}$ column. Scrambled peptides were used as a control. Representative FPLC profiles, peak square data, and statistical evaluation are shown for each treatment. The thin arrows on FPLC profiles indicate the location of molecular weight standards; from left to right: $128 \mathrm{kDa}, 44 \mathrm{kDa}$, and $17 \mathrm{kDa}$. $A$, N-terminal AANAT-specific and control (NCTRL) peptides. $\boldsymbol{B}, \mathrm{C}$-terminal AANAT-specific and control (CCTRL) peptides. C, Combination of N-terminal and C-terminal AANAT-specific peptides. ${ }^{*} p<0.05$ when compared with scrambled peptide control group; ${ }^{\dagger} p<0.05$ when compared with phospho-AANAT22-37 + AANAT195-205 group; Student-Newman-Keuls method following one-way RM-ANOVA $p<0.01$. Values are means $\pm \operatorname{SEM}(n=3)$.

light exposure, proteolytic degradation of the free enzyme causes a decrease in $V_{\max }$. In the daytime, most of the free enzyme is already degraded; hence, the lower $V_{\max }$. The remaining enzyme, mostly protected from degradation by 14-3-3, has an apparent $K_{\mathrm{m}}$ that is intermediate between that in darkness and that after acute light exposure (Fig. 1).

Previous chromatographic analyses have shown that rat pineal AANAT (Namboodiri et al., 1987) and recombinant human AANAT (Ganguly et al., 2001) separate into two peaks that also correspond to 14-3-3-complexed and free enzyme, suggesting that similar mechanisms may be operative in regulating activity of these mammalian AANATs. However, it should be noted that the high molecular weight form of AANAT has not been reported previously. In our experiments, this form was only observed in appreciable amounts in extracts of light-exposed retinas that had been treated with the proteasome inhibitor lactacystin (Fig. 6). This observation suggests that the high molecular weight AANAT may represent enzyme targeted to the proteasome and that it does not accumulate in the absence of proteasomal inhibition because of rapid degradation. This high molecular weight peak of AANAT activity separates over a broad range of apparent molecular weights, from $\sim 150$ to $>670 \mathrm{kDa}$ (Fig. $2 C)$, suggesting that it is heterogeneous, possibly representing polyubiquitinated enzyme. However, because the peak contains a $23 \mathrm{kDa}$ band of AANAT (Fig. 5), AANAT does not appear to be ubiquitinated. Rather, it may be bound to a variety of proteins, perhaps components of the proteasomal degradation pathway. Another possibility is that AANAT molecules in the high molecular weight peak may be denatured to varying degrees, altering their mobility on the gel permeation column. Alternatively, the $23 \mathrm{kDa}$ band may be AANAT that underwent deubiquitination while being concentrated and prepared for Western blot analysis.

Light induces AANAT dephosphorylation of the N-terminal PKA site in retina (Fig. 7). Phosphorylation is essential for ovine AANAT binding to 14-3-3 (Ganguly et al., 2001, 2005). Incubation of retinal extracts with the catalytic subunit of PKA or with 8-Br-cAMP favors the formation of the AANAT/14-3-3 complex (Fig. 10), probably by phosphorylation of Thr-29 and Ser-203. Using synthetic peptides corresponding to the $\mathrm{N}$ - and C-terminal phosphorylation sites, we observed that only peptides containing phosphorylated PKA sites were able to dissociate retinal AANAT/14-3-3 complex and initiate AANAT degradation (Fig. 9). The effect of phosphorylated C-terminal peptide alone was much less prominent when compared with N-terminal peptide, the ability of which to dissociate the complex was potentiated by the phosphorylated C-terminal peptide when used in combination. Thus, phospho-Thr-29 has more impact on the affinity of AANAT for 14-3-3 than does phospho-Ser-203.

Phosphorylation of AANAT promotes binding to 14-3-3, protecting the enzyme from degradation. In addition, we have shown that 14-3-3 protects AANAT from dephosphorylation, as suggested previously (Ganguly et al., 2001). Dissociation of the AANAT/14-3-3 complex in retinal extracts with R18 or phosphate buffer resulted in complete dephosphorylation of the enzyme, even at $4^{\circ} \mathrm{C}$ (Fig. 8 ). 
Incubation of AANAT with ATP/ $\mathrm{Mg}^{2+}$ alone, without cAMP, caused an increase in free AANAT activity but not in the 14-3-3 complexed enzyme (Fig. 7). The mechanism for this effect is unknown. Nucleotide triphosphates have been shown to augment rat pineal AANAT activity (Namboodiri et al., 1979). These effects could be caused by allosteric modulation of the enzyme, or ATP may serve as a substrate for cyclic nucleotideindependent kinases present in retinal supernatant that phosphorylate AANAT and protect the enzyme from proteolysis.

It is likely that the acute inhibitory effect of light on retinal AANAT activity is mediated by shutting off the cAMP/PKA pathway. Photoreceptor cAMP is regulated by light exposure, which decreases levels of the second messenger (Orr et al., 1976; DeVries et al., 1978; Cohen, 1982). In photoreceptor-enriched chick retinal cell cultures, light treatment at night dramatically decreased the cAMP levels and AANAT activity, and the effects of light on AANAT activity were reversed by incubating the cells with $8 \mathrm{Br}$-cAMP or lactacystin (Ivanova and Iuvone, 2003).

Understanding the role of 14-3-3 in the regulation of AANAT helps to explain some results and discrepancies observed previously. The ability of phosphate to dissociate AANAT from 14-3-3, coupled with the high $V_{\max }$ of the free enzyme, provides a molecular mechanism for the previous observation that high molarity phosphate causes dramatic increases of AANAT activity from chicken retina and pineal gland (Hamm and Menaker, 1981).

A previous study reported that lactacystin completely blocked light-evoked suppression of AANAT activity in chicken retina while only partially suppressing the reduction of AANAT protein (Iuvone et al., 2002). It is clear now that light treatment releases AANAT from the complex with 14-3-3; the high $V_{\max }$ of free enzyme resulted in preservation of AANAT activity despite the partial degradation of the protein after light exposure.

Collectively, our observations and those in the literature support the following working model for regulation of AANAT in chicken photoreceptor cells (Fig. 11). AANAT is synthesized as a monomeric enzyme. This occurs to a greater extent at night than during the day because of the circadian control of aanat transcript level (Bernard et al., 1997). During synthesis, the monomeric enzyme (peak 3 ) quickly establishes a dynamic equilibrium with the pool of 14-3-3 bound enzyme (peak 2). This equilibrium is strongly shifted toward complex formation in the presence of cAMP; however, a small portion of enzyme is always free. This assumption is supported by the finding that a significant portion of AANAT is bound to 14-3-3 during the day (Fig. 6). The fraction of total AANAT that is free is susceptible to dephosphorylation, leading to its conversion to a transient state (peak 1) that is quickly degraded by the proteasome. However, at night and in darkness, a significant amount of the free enzyme remains phosphorylated because of high levels of cAMP and PKA activity, and

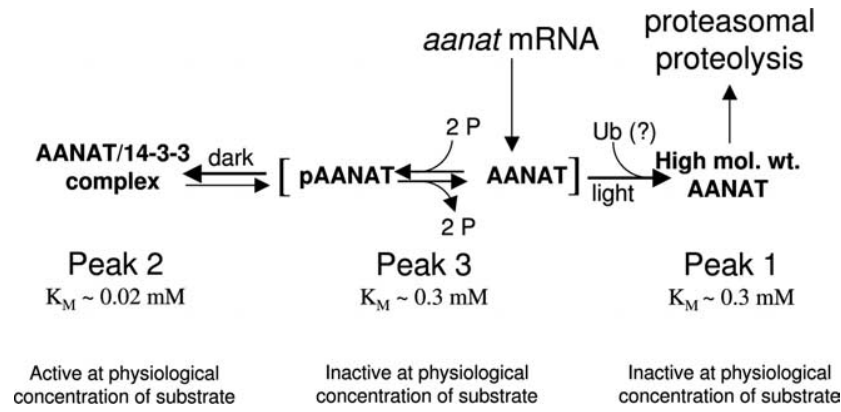

Figure 11. Working model for the regulation of AANAT in photoreceptor cells. See Discussion for details.

total amount of AANAT (14-3-3 bound and unbound) remains high. Light decreases cAMP, which allows free phosphorylated AANAT to be dephosphorylated, promoting a rapid shift in bound/free ratio to the free form, resulting in degradation of the free enzyme. Thus, small light-induced changes in phosphatase/kinase activities may result in large and rapid changes in AANAT protein. The association with 14-3-3 significantly lowers the $K_{\mathrm{m}}$ for substrate, activating it at physiological concentrations of serotonin. Thus, it is probable that the size of the pool of 14-3-3 bound enzyme is the primary determinant of the rate of $\mathrm{N}$-acetylserotonin formation 
and, consequently, the synthesis of melatonin, a neuromodulator that regulates day/night changes in retinal physiology.

\section{References}

Aitken A, Baxter H, Dubois T, Clokie S, Mackie S, Mitchell K, Peden A, Zemlickova E (2002) Specificity of 14-3-3 isoform dimer interactions and phosphorylation. Biochem Soc Trans 30:351-360.

Bernard M, Iuvone PM, Cassone VM, Roseboom PH, Coon SL, Klein DC (1997) Avian melatonin synthesis: photic and circadian regulation of serotonin $N$-acetyltransferase mRNA in the chicken pineal gland and retina. J Neurochem 68:213-224.

Besharse JC, Iuvone PM, Pierce ME (1988) Regulation of rhythmic photoreceptor metabolism: a role of post-receptoral neurons. Prog Retin Res 7:21-61.

Binkley S, Klein DC, Weller JL (1976) Pineal serotonin N-acetyltransferase activity: protection of stimulated activity by acetyl-CoA and related compounds. J Neurochem 26:51-55.

Cahill GM, Besharse JC (1992) Light-sensitive melatonin synthesis by Xenopus photoreceptors after destruction of the inner retina. Vis Neurosci $8: 487-490$.

Chaurasia SS, Rollag MD, Jiang G, Hayes WP, Haque R, Natesan A, Zatz M, Tosini G, Liu C, Korf HW, Iuvone PM, Provencio I (2005) Molecular cloning, localization and circadian expression of chicken melanopsin (Opn4): differential regulation of expression in pineal and retinal cell types. J Neurochem 92:158-170.

Chong NW, Bernard M, Klein DC (2000) Characterization of the chicken serotonin $\mathrm{N}$-acetyltransferase gene. Activation via clock gene heterodimer/E box interaction. J Biol Chem 275:32991-32998.

Cohen AI (1982) Increased levels of $3^{\prime}, 5^{\prime}$-cyclic adenosine monophosphate induced by cobaltous ion or 3-isobutylmethylxanthine in the incubated mouse retina: evidence concerning location and response to ions and light. J Neurochem 38:781-796.

DeVries GW, Cohen AI, Hall IA, Ferrendelli JA (1978) Cyclic nucleotide levels in normal and biologically fractionated mouse retina: effects of light and dark adaptation. J Neurochem 31:1345-1351.

Falcon J, Collin JP (1991) Pineal-retinal relationships: rhythmic biosynthesis and immunocytochemical localization of melatonin in the retina of the pike (Esox lucius). Cell Tissue Res 265:601-609.

Falcon J, Galarneau KM, Weller JL, Ron B, Chen G, Coon SL, Klein DC (2001) Regulation of arylalkylamine $N$-acetyltransferase-2 (AANAT2, EC 2.3.1.87) in the fish pineal organ: evidence for a role of proteasomal proteolysis. Endocrinology 142:1804-1813.

Fu H, Subramanian RR, Masters SC (2000) 14-3-3 proteins: structure, function, and regulation. Annu Rev Pharmacol Toxicol 40:617-647.

Ganguly S, Gastel JA, Weller JL, Schwartz C, Jaffe H, Namboodiri MA, Coon SL, Hickman AB, Rollag M, Obsil T, Beauverger P, Ferry G, Boutin JA, Klein DC (2001) Role of a pineal cAMP-operated arylalkylamine $\mathrm{N}$-acetyltransferase/14-3-3-binding switch in melatonin synthesis. Proc Natl Acad Sci USA 98:8083-8088.

Ganguly S, Weller JL, Ho A, Chemineau P, Malpaux B, Klein DC (2005) Melatonin synthesis: 14-3-3-dependent activation and inhibition of arylalkylamine $N$-acetyltransferase mediated by phosphoserine-205. Proc Natl Acad Sci USA 102:1222-1227.

Gastel JA, Roseboom PH, Rinaldi PA, Weller JL, Klein DC (1998) Melatonin production: proteasomal proteolysis in serotonin $N$-acetyltransferase regulation. Science 279:1358-1360.

Gern WA, Ralph CL (1979) Melatonin synthesis by the retina. Science 204:183-184.

Green CB, Besharse JC (2004) Retinal circadian clocks and control of retinal physiology. J Biol Rhythms 19:91-102.

Hamm HE, Menaker M (1980) Retinal rhythms in chicks: circadian variation in melantonin and serotonin $\mathrm{N}$-acetyltransferase activity. Proc Natl Acad Sci USA 77:4998-5002.

Hamm HE, Menaker M (1981) Pineal and retinal serotonin $N$-acetyltransferase activity: modulation by phosphate. J Neurochem 37:1567-1572.

Haque R, Chaurasia SS, Wessel III JH, Iuvone PM (2002) Dual regulation of cryptochrome 1 mRNA expression in chicken retina by light and circadian oscillators. NeuroReport 13:2247-2251.

Iuvone PM, Brown AD, Haque R, Weller J, Zawilska JB, Chaurasia SS, Ma M, Klein DC (2002) Retinal melatonin production: role of proteasomal proteolysis in circadian and photic control of arylalkylamine $\mathrm{N}$-acetyltransferase. Invest Ophthalmol Vis Sci 43:564-572.

Iuvone PM, Tosini G, Pozdeyev N, Haque R, Klein DC, Chaurasia SS (2005) Circadian clocks, clock networks, arylalkylamine $N$-acetyltransferase, and melatonin in the retina. Prog Retin Eye Res 24:433-456.

Ivanova TN, Iuvone PM (2003) Circadian rhythm and photic control of cAMP level in chick retinal cell cultures: a mechanism for coupling the circadian oscillator to the melatonin-synthesizing enzyme, arylalkylamine $N$-acetyltransferase, in photoreceptor cells. Brain Res 991:96-103.

Klein DC (2004) The 2004 Aschoff/Pittendrigh lecture: theory of the origin of the pineal gland-a tale of conflict and resolution. J Biol Rhythms 19:264-279.

Kockel L, Vorbruggen G, Jackle H, Mlodzik M, Bohmann D (1997) Requirement for Drosophila 14-3-3 zeta in Raf-dependent photoreceptor development. Genes Dev 11:1140-1147.

Lerner AB, Case JD, Takahashi Y, Lee TH, Mori W (1958) Isolation of melatonin, the pineal gland factor, that lightens melanocytes. J Am Chem Soc 80:2587.

Lowry OH, Rosebrough NJ, Farr AL, Randall RJ (1951) Protein measurement with the folin phenol reagent. J Biol Chem 193:265-275.

Moore BW, Perez VJ (1967) Specific acidic proteins of the nervous system. In: Physiological and biochemical aspects of nervous integration (Carlson FD, ed), pp 343-359. New York: Prentice-Hall.

Nakano K, Chen J, Tarr GE, Yoshida T, Flynn JM, Bitensky MW (2001) Rethinking the role of phosducin: light-regulated binding of phosducin to 14-3-3 in rod inner segments. Proc Natl Acad Sci USA 98:4693-4698.

Namboodiri MA, Nakai C, Klein DC (1979) Effects of selected treatments on stability and activity of pineal serotonin $\mathrm{N}$-acetyltransferase. J Neurochem 33:807-810.

Namboodiri MA, Brownstein MJ, Weller J, Voisin P, Klein DC (1987) Multiple forms of arylalkylamine $\mathrm{N}$-acetyltransferases in the rat pineal gland: purification of one molecular form. J Pineal Res 4:235-246.

Niki T, Hamada T, Ohtomi M, Sakamoto K, Suzuki S, Kako K, Hosoya Y, Horikawa K, Ishida N (1998) The localization of the site of arylalkylamine $\mathrm{N}$-acetyltransferase circadian expression in the photoreceptor cells of mammalian retina. Biochem Biophys Res Commun 248:115-120.

Obsil T, Ghirlando R, Klein DC, Ganguly S, Dyda F (2001) Crystal structure of the 14-3-3zeta:serotonin $N$-acetyltransferase complex. A role for scaffolding in enzyme regulation. Cell 105:257-267.

Orr HT, Lowry OH, Cohen AI, Ferrendelli JA (1976) Distribution of $3^{\prime}: 5^{\prime}$ cyclic AMP and $3^{\prime}: 5^{\prime}$-cyclic GMP in rabbit retina in vivo: selective effects of dark and light adaptation and ischemia. Proc Natl Acad Sci USA 73:4442-4445.

Petosa C, Masters SC, Bankston LA, Pohl J, Wang B, Fu H, Liddington RC (1998) 14-3-3zeta binds a phosphorylated Raf peptide and an unphosphorylated peptide via its conserved amphipathic groove. J Biol Chem 273:16305-16310.

Roseboom PH, Weller JL, Babila T, Aitken A, Sellers LA, Moffett JR, Namboodiri MA, Klein DC (1994) Cloning and characterization of the epsilon and zeta isoforms of the 14-3-3 proteins. DNA Cell Biol 13:629-640.

Schomerus C, Korf HW, Laedtke E, Weller JL, Klein DC (2000) Selective adrenergic/cyclic AMP-dependent switch-off of proteasomal proteolysis alone switches on neural signal transduction: an example from the pineal gland. J Neurochem 75:2123-2132.

Thomas KB, Zawilska J, Iuvone PM (1990) Arylalkylamine (serotonin) $\mathrm{N}$-acetyltransferase assay using high-performance liquid chromatography with fluorescence or electrochemical detection of $N$-acetyltryptamine. Anal Biochem 184:228-234.

Wang B, Yang H, Liu YC, Jelinek T, Zhang L, Ruoslahti E, Fu H (1999) Isolation of high-affinity peptide antagonists of 14-3-3 proteins by phage display. Biochemistry 38:12499-12504.

Zatz M, Gastel JA, Heath III JR, Klein DC (2000) Chick pineal melatonin synthesis: light and cyclic AMP control abundance of serotonin $N$-acetyltransferase protein. J Neurochem 74:2315-2321.

Zheng W, Zhang Z, Ganguly S, Weller JL, Klein DC, Cole PA (2003) Cellular stabilization of the melatonin rhythm enzyme induced by nonhydrolyzable phosphonate incorporation. Nat Struct Biol 10:1054-1057.

Zheng W, Schwarzer D, Lebeau A, Weller JL, Klein DC, Cole PA (2005) Cellular stability of serotonin $N$-acetyltransferase conferred by phosphonodifluoromethylene alanine (Pfa) substitution for Ser-205. J Biol Chem 280:10462-10467. 